\title{
Reconfiguration point decision method based on dynamic complexity for reconfigurable manufacturing system (RMS)
}

\author{
Sihan Huang ${ }^{1} \cdot$ Guoxin Wang $^{1} \cdot$ Xiwen Shang $^{1} \cdot \operatorname{Yan~Yan}^{1}$
}

Received: 2 September 2016 / Accepted: 28 February 2017 / Published online: 9 March 2017

(C) The Author(s) 2017. This article is published with open access at Springerlink.com

\begin{abstract}
To address the problem of how to identify the best time to implement reconfiguration for the reconfigurable manufacturing system (RMS), a dynamic complexity-based RMS reconfiguration point decision method is proposed. This method first identifies factors that affect RMS dynamic complexity (including both positive and negative complexity) at the machine tool and manufacturing cell levels. Next, based on information entropy theory, a quantitative model for RMS dynamic complexity is created, which is solved via state probability analysis for processing capability and the processing function. This model is combined with cusp catastrophe theory to establish an RMS reconfiguration decision model. Both positive and negative complexity are control variables for cusp catastrophe. Cusp catastrophe's state condition is used to identify RMS state catastrophe at the final stage of production. This catastrophe point is the RMS reconfiguration point. Finally, the case study result shows that this method can effectively identify the RMS state catastrophe moment so that system reconfiguration is implemented promptly to improve RMS's responsiveness to the market.
\end{abstract}

Keywords Reconfigurable manufacturing system - Reconfiguration point decision - Information entropy - Cusp catastrophe $\cdot$ Dynamic complexity

\footnotetext{
Guoxin Wang

wangguoxin@bit.edu.cn

1 Industrial Engineering Research Institute, School of Mechanical Engineering, Beijing Institute of Technology, Beijing 100081, China
}

\section{Introduction}

In 2010, the German government proposed the concept of Industry 4.0 in its report "High-Tech Strategy (2010)". That report marked the prologue to the fourth Industrial Revolution, which is dominated by intelligent manufacturing. Lee Jie, Professor and Distinguished Chair at The University of Cincinnati (US) and director of the National Science Foundation's Intelligent Maintenance System Industry/University Cooperative Center, believes that there are three pillars in the development of Industry 4.0. One of them is that "manufacturing systems, including the machine tool itself, are able to make adjustments automatically and promptly according to differences in the processed product and changes in processing condition, and possess the so-called 'self-reflection' capability" (Lee et al. 2015). The RMS reconfiguration capability satisfies the requirement of intelligent manufacturing and is a critical component in future intelligent manufacturing environments. Mehrabi et al. (2000a, 2002) provide an outlook for RMS research focus and future development and suggest that RMS is a desirable next step in the evolution of production systems. The reconfiguration moment decision is essential for RMS to be the backbone of intelligent manufacturing; it is also a critical and fundamental problem waiting for a solution on the road to intelligent manufacturing.

RMS is a manufacturing system with fast response capability. Its architecture, hardware and software can be reassembled and adjusted to rapidly change the system function to respond to impacts from market fluctuation, technology innovation and policy change (Koren et al. 1999; Koren 2013; Mehrabi et al. 2000b). Because of its huge market potential, researchers have studied various aspects of RMS, including reconfigurability (Wang 2000), system layout planning (Goyal et al. 2012; Wu et al. 2007), system performance analysis (Cai 2004), part-family formation (Wang et al. 2016a; 
Goyal et al. 2013; Gupta et al. 2013; Hasan et al. 2014) and reconfiguration schemes evaluation (Wang et al. 2016b). Zhao et al. (2000a, b, 2001a,b) provide a stochastic RMS model that includes a framework, optimal configuration, optimal selection policy and performance measurement. Bi et al. (2008) provides an overview of RMS. Renzi et al. (2014) elaborate the advantages of an RMS in terms of cost and efficiency and conduct an in-depth analysis of key technologies in RMS. Although RMS has excellent architecture that can respond to rapid changes in market demand (Wang 2000), the issue of when to implement such a change is a complex and difficult decision problem. The decision about when to implement RMS reconfiguration should consider various production factors such as cost, time and order; a conclusion based on the analysis of any single factor is not convincing. Therefore, this paper has analyzed the RMS reconfiguration point from the system's perspective. Researchers have conducted comprehensive analyzing manufacturing systems via system state. Rao (2006) provide a quantitative description of manufacturing system state complexity from the perspective of information theory; they analyze the manufacturing system's static complexity and dynamic complexity and create an information entropy-based static and dynamic complexity measurement model. To address deficiencies in the manufacturing system architecture complexity modeling method, Duan (2012) analyze the effect of the intermediate buffer zone state on the manufacturing system state; they also employ the universal generating function and information entropy theory to create and evaluate a model for non-serial manufacturing system complexity. Smart et al. (2013) study the manufacturing system's dynamic complexity based on improved information entropy theory; their main focus is an analysis of equipment and queue state in the production process and an elaboration of the relationship between system complexity and system operation. Zhang (2011) analyze the manufacturing system dissipation structure; create a manufacturing system entropy variation model based on information entropy theory; analyze the relationship between the manufacturing system control variable and the state variable based on catastrophe theory; and perform a quantitative analysis of system dissipation structure formation. The aforementioned manufacturing state analysis is only based on a part state or the machine tool state, whereas the manufacturing system state is determined by interaction between parts and machine tools. The part type demands a specific processing function from the manufacturing system; the part quantity demands a corresponding processing capability from the manufacturing system. As provider of processing function and processing capability, the machine tool provides one or more processing functions and processing capabilities of specific quantities. To summarize, the analysis of manufacturing system complexity in terms of processing function and processing capability fully describes the interaction between parts and machine tools, reflects the nature of the manufacturing system state, and thus provides evidence for the RMS reconfiguration decision.

Therefore, this paper proposes a dynamic complexitybased RMS reconfiguration point decision method. Based on an analysis of the RMS reconfiguration mechanism, the effect of system processing function and processing capability on system complexity is investigated and an information entropy-based RMS complexity quantitative model is created. This model is combined with a cusp catastrophe to analyze system state variation and identify the RMS state catastrophe moment.

\section{RMS reconfiguration mechanism and problem analysis}

After RMS construction is completed, a running-in period is required; that period is called the ramp-up time (Rösiö and Säfsten 2013). During this period, system stability is relatively low, and problems such as machine failure and substandard products are likely to emerge. After a period of tuning and running-in, problems in the new system are gradually fixed; the system operates in a highly efficient and stable state and is capable of high-quality, high-yield and low-cost production. This period is called the stable production period. As RMS continues its operation, because of random internal and external factors such as machine tool failure and new production order queue-jumping, the system fails to provide sufficient processing functions and capability. The leads to delayed order delivery, more difficult scheduling, declined productivity and chaos in production, which prompts system reconfiguration. At this moment, the system runs into the end stage of production. RMS stops production at the right moment and enters the reconfiguration period. Machine tools and components are added, rearranged, removed and adjusted to reconfigure the RMS processing function and processing capability. When reconfiguration is completed, the RMS processing function and processing capability are updated; system capability is able to meet new market demand; and RMS starts a new round of production cycles. In this cyclic process, the quantitative accumulation of machine tool blocks and failures leads to a qualitative change in the system state, or state catastrophe. After a catastrophe, system-level reconfiguration is required and scheduling alone is unable to solve system problems. The key to this process is to identify the catastrophe moment, which is also this paper's focus. Every RMS reconfiguration updates system capability and thus always maintains a relatively high market response capability and productivity. Figure 1 shows the RMS reconfiguration mechanism and implementation process. That diagram shows that after a temporary ramp-up time, RMS can provide processing function 


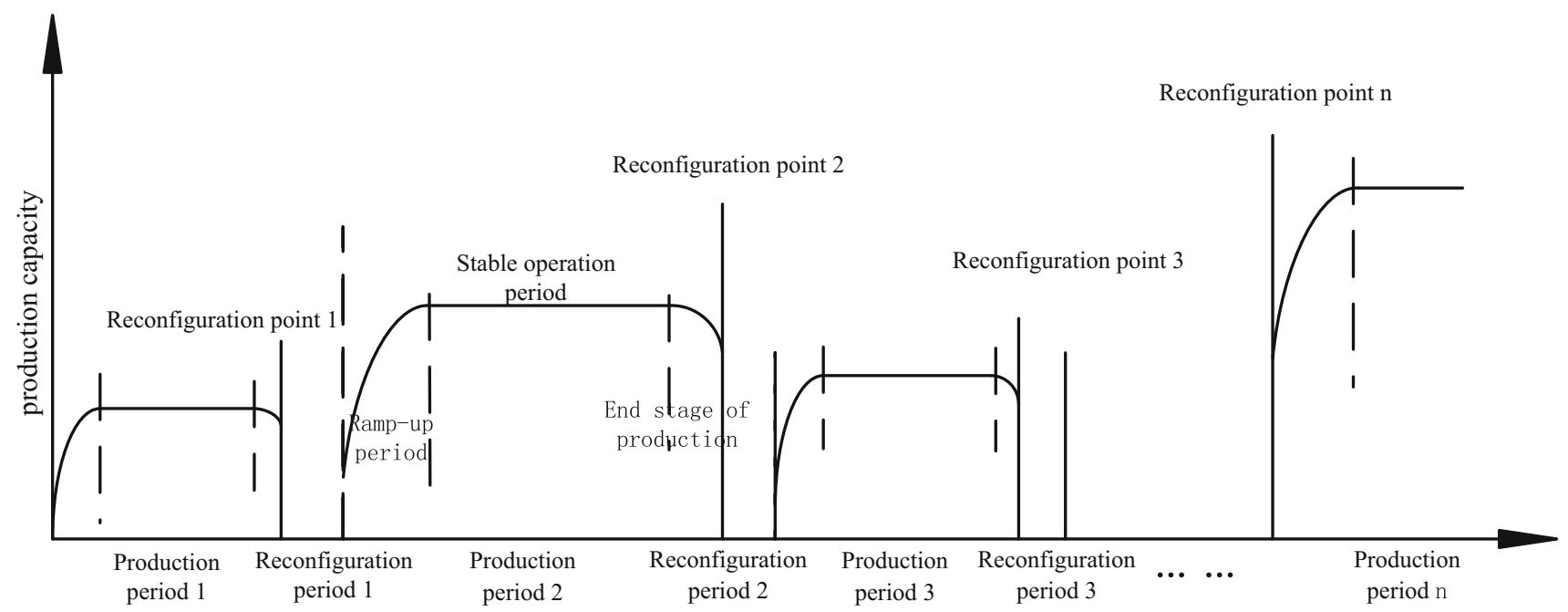

Fig. 1 RMS reconfiguration mechanism and implementation process

and processing capability for current production requirements, and the system is experiencing stable production. At the end stage of production, because of the combined effect of system internal and external factors, three problems arise in the RMS processing function and processing capability system: (1) the processing-function requirement is met, but the processing capability is inadequate; (2) processing capability is adequate, but the processing function is inadequate; and (3) both processing function and processing capability are inadequate. At this moment, system operation efficiency declines and system production capacity gradually declines, leading to delayed order delivery. At a certain moment, processing function and processing capability deficiency leads to manufacturing system state catastrophe; the system enters a reconfiguration period to rebuild processing function and processing capability and then moves to the next production cycle.

The RMS state is the result of interaction between processing function and processing capability driven by orders. Factors such as order fluctuation and machine tool failure lead to variation in processing function and processing capability, resulting in system state fluctuation. RMS complexity measures fluctuation in a system's processing function and processing capability. System complexity variation reflects variations in system state stability. In a normal situation, as the system processing function gradually increases, the processing function and processing capability fluctuation are relatively stable, system complexity gradually increases, and system state stability gradually increases. At the end stage of production, the system processing function gradually becomes saturated, the processing function and processing capability fluctuation increase; and system complexity continues to increase. However, system state stability demonstrates a trend of accelerated decline. Figure 2 shows the

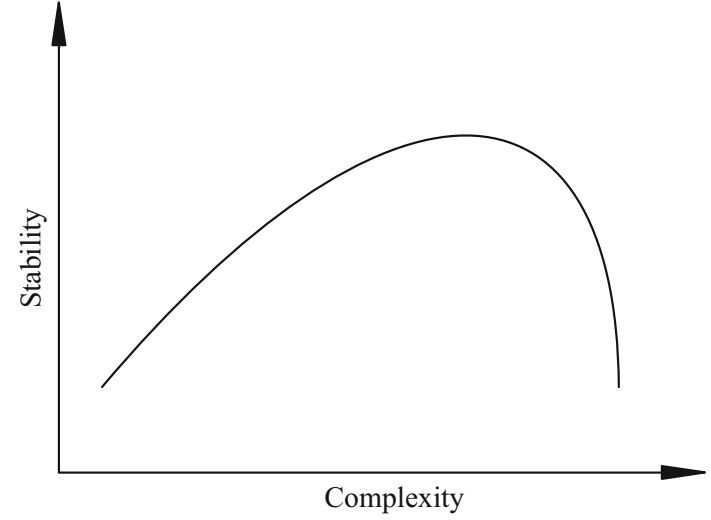

Fig. 2 Relationship between RMS complexity and stability

relationship between system complexity and system state stability (Qin 2004).

When the RMS processing function or processing capability fails to meet order demand, if reconfiguration is implemented immediately, the normal result is a relatively high cost attributable to production down time. When there are a large number of delayed orders, system reconfiguration is more difficult and costly. The decision about the reconfiguration moment becomes a critical step in RMS implementation. In other words, at the end stage of production, system complexity variation is analyzed to identify the catastrophe point of the system stable state. When system stable state catastrophe occurs, continued production normally results in a dramatic increase in cost. Moreover, if production continues until the system is in an extremely unstable state, system reconfiguration requires significantly higher cost and a much longer time. Therefore, when a system stable state catastrophe occurs, it is the best time to implement system reconfiguration. This reconfiguration moment is the RMS 
reconfiguration point. In this paper, based on information entropy, system complexity variation is analyzed quantitatively and is combined with cusp catastrophe to complete the decision about the RMS reconfiguration point.

\section{Information entropy-based quantitative analysis of RMS complexity}

\section{RMS complexity analysis}

Manufacturing system complexity variation should be considered in the RMS reconfiguration decision process. There are two categories of manufacturing system complexities: static complexity and dynamic complexity. Static complexity is primarily concerned with the production state of the manufacturing system according to its scheduling plan. However, in actual production, because of uncertain factors such as market fluctuation and machine failure, production normally deviates from the scheduling plan. Dynamic complexity describes a manufacturing system's actual operational state, which represents system state variation in actual production. Therefore, in this paper, system complexity is simplified as dynamic complexity and RMS state analysis is based on dynamic complexity.

System complexity is a measurement of system uncertainty. Information entropy (Czinner and Mena 2016; Duan 2012; Rao 2006; Tsujimura and Gen 1999; Shannon 1948) represents the amount of information contained in a system state. Information entropy accurately reflects the system complexity variation. When system complexity is very low, system uncertainty is low, which means that the system is in an ordered state. In this case, less information is required to describe the system state, and system information entropy is relatively small. However, when system complexity is very high, the system state is difficult to predict, which means that the system is in a chaotic state. In this case, more information is required to describe the system, which features relatively large information entropy. Therefore, a quantitative analysis of RMS complexity is based on information entropy theory, whose mathematical description is shown in formula (1) (Shannon 1948).

$$
\begin{gathered}
E(X)=-\sum_{i=1}^{n} p_{i} \mathrm{lb} p_{i} \\
p_{i} \geq 0 \\
\sum_{i=1}^{n} p_{i}=1
\end{gathered}
$$

where $X$ represents a system; $E(X)$ represents information contained in system $X$ or the information entropy of system $X ; p_{i}(i=1,2, \ldots, \mathrm{n})$ represents the probability of system
$X$ in the $i$-th symbol; and lb represents logarithmic to the base 2.

Based on the RMS definition, RMS's rapid-response capability is determined by each manufacturing cell's ability to adjust processing capability and processing function; i.e., RMS reconfiguration is driven by adjusting the processing capability and processing function. Therefore, the RMS processing capability and processing function are chosen as indexes to describe system complexity. At end stage of each production period, RMS processing capability and processing function state probability are analyzed to create the RMS complexity information-entropy model. In the analysis of the system processing capability state, machine tool productivity and the buffer zone state are chosen as analysis indexes; in the analysis of the system processing function state, the machine tool processing state is chosen as the analysis index.

\section{Information entropy-based RMS dynamic complexity model}

RMS is composed of several manufacturing cells. Each manufacturing cell contains numerous machine tools. Each type of machine tool is classified as the same type of processing function. The processing route establishes the relationship between processing functions. Part quantity is a test of a machine tool's processing capability; i.e., that affects a machine tool's productivity. When RMS is in operation, the machine tool is in specific state; machine tool quantity and machine tool state in the cellt directly determine cell state. Similarly, overall states of cells in the system determine the system state. Therefore, the machine tool state in the cell should be identified.

When the machine tool is in normal operation and contributes to system stability, it is in an operative/non-blocked state. When the machine tool can complete its processing task but its efficiency is declining and compromises system stability, that tool is in an operative/blocked state. When the machine tool fails to complete its production task because of environmental disturbance (such as a new order or equipment failure) and causes production to be put on hold, it is in an inoperative state. Combined fluctuation in machine tool quantity, machine tool state and cell state determines system complexity, whereas machine tool state type determines complexity type. Based on the effect of the machine tool state on system stability, system complexity is classified as positive complexity and negative complexity. This classification is combined with information entropy theory to create the system dynamic complexity quantitative model shown in formulae (2), (3) and (4):

$E_{X}=E_{p}+E_{n}$ 


$$
\begin{aligned}
E_{p}= & -\sum_{i}^{N} \sum_{j}^{G_{i}} \sum_{k}^{\left|S_{j}\right|} p_{i j k}^{1} \mathrm{lb} p_{i j k}^{1} \\
E_{n}= & \left(-\sum_{i}^{N} \sum_{j}^{G_{i}} \sum_{k}^{\left|S_{j}\right|} p_{i j k}^{2} \mathrm{lb} p_{i j k}^{2}\right) \\
& +\left(-\sum_{i}^{N} \sum_{j}^{G_{i}} \sum_{k}^{\left|S_{j}\right|} p_{i j k}^{3} \mathrm{lb} p_{i j k}^{3}\right)
\end{aligned}
$$

where, $E_{X}$ represents RMS dynamic complexity; $E_{p}$ represents RMS positive complexity; and $E_{n}$ represents RMS negative complexity. RMS dynamic complexity is weighted sum of positive complexity and negative complexity. Positive complexity is information entropy for the operative/nonblocked state; negative complexity is the sum of information entropy for the operative/blocked and inoperative states. $N$ represents the quantity of cells in RMS; $G_{i}$ represents types of parts in the $i$-th cell; and $S_{j}$ represents the processing route for the $j$-th type of part in the $i$-th cell. For instance, when a manufacturing cell contains 2 types of parts, processing routes for parts 1 and 2 are $\left\{\begin{array}{lll}a & b & c\end{array}\right\}$ and $\left\{\begin{array}{lll}a & c & d\end{array}\right\}$, respectively; next, there is $S_{1}=\left\{\begin{array}{lll}a & b & c\end{array}\right\}$ and $S_{2}=\left\{\begin{array}{lll}a & c & d\end{array}\right\}$, where $a, b, c$ and $d$ represent processing functions. Each type of processing function can contain multiple machine tools. $\left|S_{j}\right|$ represents processing route length; $p_{i j k}^{1}$ represents the probability of the operative/non-blocked state when the $j$-th type of part in the $i$-th cell calls for the $k$-th processing function in processing route $\mathrm{S}_{j} ; p_{i j k}^{2}$ represents the probability of the operative/blocked state when the $j$-th type of part in the $i$-th cell calls for the $k$-th processing function in processing route $\mathrm{S}_{j} ; p_{i j k}^{3}$ represents the probability of the inoperative state when the $j$-th type of part in the $i$-th cell calls for the $k$-th processing function in processing route $\mathrm{S}_{j}$.

The machine tool occupies three states: operative/nonblocked, operative/blocked and inoperative. The operative and inoperative states are related to the processing function. The non-blocked and blocked states are related to processing capability. Because processing function and processing capability are independent from each other, operative/inoperative states and unblock/blocked states are also independent from each other. Machine tool state probability is treated as the combined probability of two types of states: processing function and processing capability. In other words, operative/non-blocked states probability is the product of the probability of the machine tool in its operative state and the probability of the machine tool in its non-blocked state; operative/blocked state probability is the product of the probability of the machine tool in its operative state and the probability of the machine tool in its blocked state. To summarize, the solution for the probability of the machine tool's three states is shown in formula (5):

$$
\left\{\begin{array}{l}
p_{i j k}^{1}=p_{i j k}^{K} \cdot p_{i j k}^{C} \\
p_{i j k}^{2}=p_{i j k}^{K} \cdot p_{i j k}^{D} \\
p_{i j k}^{3}=p_{i j k}^{B}
\end{array}\right.
$$

where $p_{i j k}^{K}$ represents the probability of the operative state when the $j$-th type of part in the $i$-th cell calls for the $k$-th processing function in processing route $\mathrm{S} j ; p_{i j k}^{C}$ represents the probability of the non-blocked state when the $j$-th type of part in the $i$-th cell calls for the $k$-th processing function in processing route $\mathrm{S} j ; p_{i j k}^{D}$ represents the probability of the blocked state when the $j$-th type of part in the $i$-th cell calls for the $k$-th processing function in processing route $\mathrm{S} j$; and $p_{i j k}^{B}$ represents the probability of the inoperative state when the $j$-th type of part in the $i$-th cell calls for the $k$-th processing function in processing route $\mathrm{S} j$.

\section{Analysis of processing function probability}

Each machine tool may provide multiple processing functions; i.e., the relationship between machine tool and processing function is $1: 1$ or $1: n$. The same machine tool may provide different processing functions during the different processing stages of a part. Therefore, machine tools with similar processing functions in a cell are classified as the same type of processing function. The relationship between machine tools and processing functions is shown in formula (6).

$\sum_{j=1}^{n} D_{i j} \geq N_{i}$

where $N_{i}$ represents the total number of machine tools in the $i$-th manufacturing cell in RMS; $n$ represents the types of processing functions in the manufacturing cell; and $D_{i j}$ represents the quantity of machine tools with the $j$-th type of processing function in the $i$-th manufacturing cell.

The machine tool is in one of the four operation states: idle state, processing state, adjustment state (maintenance state) and failure state. This section only focuses on whether or not a cell satisfies the processing-function requirementi.e., whether the corresponding machine tool provides this function. Because a machine tool in the adjustment state or failure state fails to satisfy the operation condition, the two are classified as a single type: the failure state. Therefore, the machine tool state in this paper includes the processing state, the failure state and the idle state. During operation, when the machine tool is in the failure state, that means all of this machine tool's processing functions are in the inoperative state. In other words, when all of the machine tools providing a specific type of processing function are in the failure state, that processing function is inoperative. When the machine tool is in the operation state, it only provides a 


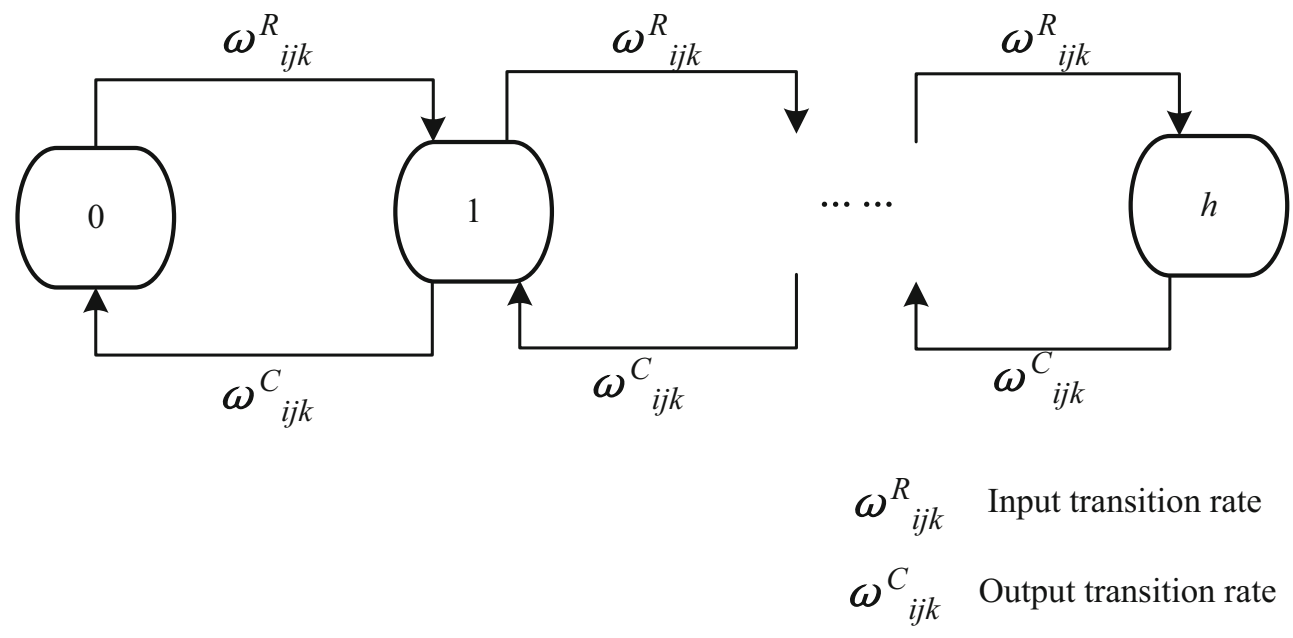

Fig. 3 Buffer zone state transition

single processing function and the other processing functions of the machine tool are all in the inoperative state. Therefore, for parts, even when machine tools with the corresponding processing function in the processing route are in the operative state, if the provided processing function is not what is required by this part, then the processing function required by this part is inoperative. To summarize, the probability of whether or not the processing function is operative is calculated via formulae (7) and (8):

$p_{i j k}^{B}=\prod_{m=1}^{M_{k}}\left(p_{i j k m}^{G}+p_{i j k m}^{\bar{J}}\right)$

$p_{i j k}^{K}=1-p_{i j k}^{B}$

where $p_{i j k m}^{G}$ represents failure probability of the $m$-th machine tool with the $k$-th type of processing function for the $j$-th type of part in the $i$-th cell; $p_{i j k m}^{\bar{J}}$ represents the probability of the $m$-th machine tool with the $k$ type of processing function for the $j$-th type of part in the $i$-th cell that is in the operative state but fails to provide the processing function required by this type of part; and $M_{k}$ represents the quantity of machine tools with the $k$-th processing function. Both $P_{i j k}^{G}$ and $P_{i j k}^{\bar{J}}$ are represented as state time frequency. Machine tool state duration in a specific period is collected, and the frequency of each state is calculated as ratio of state duration to overall time.

To summarize, the probability analysis for the processing function is completed, which includes the probability of the processing function in the operative state and the probability of the processing function in the inoperative state.

\section{Probability analysis of processing capability}

According to the analysis in "Analysis of processing function probability" section, when the processing function is in the operative state, the non-blocked and blocked states of the relevant machine tool (currently, the machine tool for part processing is determined; i.e., the relationship between the processing function and the part is limited to 1:1) should be further analyzed to obtain the processing capability state. When the buffer zone before the machine tool has stock and the buffer zone after the machine tool has vacancy, the machine tool is in the non-blocked state; when the buffer zone before the machine tool has no stock or the buffer zone after the machine tool has no vacancy, the machine tool is in the blocked state. The buffer zone state represents the machine tool state; therefore, states of buffer zones before and after the machine tool are analyzed to calculate the probability of processing capability, as shown in formulae (9) and (10):

$p_{i j k}^{C}=\left(1-p_{i j k}^{Q}\right) \times\left(1-p_{i j k}^{H}\right)$

$p_{i j k}^{D}=1-p_{i j k}^{C}$

where $p_{i j k}^{Q}$ represents the probability that the buffer zone before the machine tool has no stock; and $p_{i j k}^{H}$ represents the probability that the buffer zone after the machine tool has no vacancy.

Buffer zone probability is analyzed further. Assume that the buffer zone capacity is $h$. Then, from empty to full stock, there are $h+1$ states. When the machine tool before the buffer zone completes the processing of a part, the buffer zone state moves backward one cell; i.e., the previous machine tool's productivity is the buffer zone's input transition rate. When the machine tool after the buffer zone starts to process a part, the buffer zone state moves forward one cell; i.e., the subsequent machine tool's productivity is the buffer zone's output transition rate. The buffer zone state transition process is shown in Fig. 3.

Based on the above analysis, the state transition equation is constructed as shown in formula (11): 


$$
\begin{aligned}
& \left\{\begin{array}{c}
-w_{i j k}^{R} p_{i j k}^{0}+w_{i j k}^{C} p_{i j k}^{1}=0 \\
w_{i j k}^{R} p_{i j 1}^{0}-\left(w_{i j k}^{R}+w_{i j k}^{C}\right) p_{i j k}^{1}+w_{i j k}^{C} p_{i j k}^{2}=0 \\
\vdots \\
\vdots \\
-w_{i j k}^{R} p_{i j k}^{h-1}+w_{i j k}^{C} p_{i j k}^{h}=0
\end{array}\right. \\
& \sum_{l=0}^{h} p_{i j k}^{l}=1
\end{aligned}
$$

where $p_{i j k}^{l}$ represents the probability of the buffer zone in the $l$-th state; when $l=0, p_{i j k}^{0}=p_{i j k}^{Q}$; i.e., the probability of a buffer zone with no stock; when $l=h, p_{i j k}^{h}=p_{i j k}^{H}$; i.e., the probability of a buffer zone with no vacancy; $w_{i j k}^{R}$ represents the productivity of the machine tool before the buffer zone; and $w_{i j k}^{C}$ represents the productivity of the machine tool after the buffer zone; i.e., the productivity of the $k$-th processing function for the $j$-th part in the $i$-th cell.

The buffer zone state transition equation set (11) is solved to obtain the probability of the buffer zone state, as shown in formula (12):

$p_{i j k}^{l}= \begin{cases}\frac{\left(\frac{w_{i j k}^{R}}{w_{i j k}^{C}}\right)^{l} \cdot\left(1-\frac{w_{i j k}^{R}}{w_{i j k}^{C}}\right)}{1-\left(\frac{w_{i t r}}{w_{i j k}^{C}}\right)^{h+1}} & w_{i j k}^{R} \neq w_{i j k}^{C} \\ \frac{1}{1+h} & w_{i j k}^{R}=w_{i j k}^{C}\end{cases}$

When the buffer zone before the machine tool has no stock, there is $1=0$ in formula (12); the probability of a buffer zone before the machine tool with no stock is shown in formula (13).

$p_{i j k}^{Q}= \begin{cases}\frac{\left(1-\frac{w_{i j k}^{R}}{w_{i j k}^{C}}\right)}{1-\left(\frac{w_{i t r}}{w_{i j k}^{C}}\right)^{h+1}} & w_{i j k}^{R} \neq w_{i j k}^{C} \\ \frac{1}{1+h} & w_{i j k}^{R}=w_{i j k}^{C}\end{cases}$

When the buffer zone after the machine tool has no vacancy, there is $l=h$ in formula (12); the probability of a buffer zone after a machine tool with no vacancy is shown in formula (14).

$p_{i j k}^{Q}= \begin{cases}\frac{\left(\frac{w_{i j k}^{R}}{w_{i j k}^{C}}\right)^{l} \cdot\left(1-\frac{w_{i j k}^{R}}{w_{i j k}^{C}}\right)}{1-\left(\frac{w_{i t r}}{w_{i j k}^{C}}\right)^{h+1}} & w_{i j k}^{R} \neq w_{i j k}^{C} \\ \frac{1}{1+h} & w_{i j k}^{R}=w_{i j k}^{C}\end{cases}$

To summarize, the probability analysis of processing capability is complete, and both non-blocked and blocked states are calculated and analyzed.

\section{Cusp catastrophe model-based RMS reconfiguration point identification}

Various complexity components control system operation and determine system stability. When positive complexity of a maintenance system's stability is predominant, the system has relatively high stability and productivity. When the negative complexity component that leads to system instability is predominant, system stability significantly declines, which could result in system state catastrophe and trigger system reconfiguration. Analysis shows that during operation, RMS will experience state catastrophe because of internal and external factors such as new orders and machine tool failure. Catastrophe theory, which was proposed by French mathematician THOM in 1972, is a universal method used to investigate transition, discontinuity and catastrophe. In catastrophe theory, the system potential function is the study object, which comprises the state variable and external control parameter; the critical point of the system balance state is calculated from the potential function (Barunik and Vosvrda 2009; Chow et al. 2012; Dou and Ghose 2006; Hu and Xia 2015; Sethi and King 1998; Thom et al. 1975; Saunders 1980). Therefore, in this paper, RMS dynamic complexity is analyzed and combined with catastrophe theory to calculate and analyze the reconfiguration point.

In research by THOM, when there are no more than four control variables, no more than two types of states, and a maximum of seven types of basic catastrophe models. Four common types of catastrophe models are the folded catastrophe, the cusp catastrophe, the fork-tailed catastrophe and the butterfly catastrophe. The cusp catastrophe state variation is shown in Fig. 4. The diagram shows a folded catastrophe manifold in which the surface top lobe and bottom lobe are in stable balanced states, where the intermediate lobe is in an unstable balanced state. This diagram shares the same characteristic as the RMS implementation process in Fig. 1, in which each production period contains a stable operation period and an unstable ramp-up time. In an ideal situation, the RMS state varies stably along $A B$, and the system is in a dynamic balanced state. In actual production, because there are uncontrollable factors such as order change and machine tool failure, the system state varies along CD. After a period of stable operation, RMS state catastrophe occurs; i.e., catastrophe occurs at point $\mathrm{E}$ and the system moves from the production period to the reconfiguration period. After the system reconfiguration is completed, the new production period starts; after running-in during the ramp-up time, the new stable operation period begins. This process is called the RMS cycle. System dynamic complexity dynamically reflects this cycle; positive complexity and negative complexity are two control variables that balance each other and maintain the system state. When positive complexity dominates, the system is in stable operation period; when negative complexity 


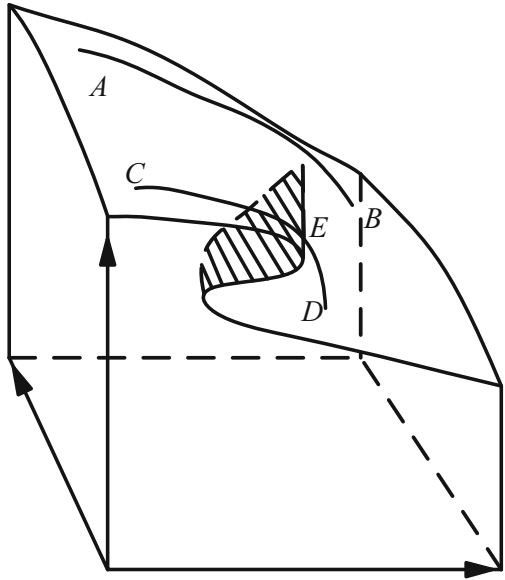

Fig. 4 Cusp catastrophe

dominates, system state catastrophe occurs; when positive complexity and negative complexity are balanced, the system is either in a ramp-up time or at the end stage of production. If the system is in a ramp-up time, positive complexity gradually dominates; if it is at the end stage of production, negative complexity gradually dominates.

Based on the above analysis, as production progresses, the gradual wearing of the machine tool results in system state change. The functional relation formula for the machine tool state and system state is expanded in the Taylor series up to the 5 th power, as shown in formula (15). Because the highest power of cusp catastrophe's state function is 4th, the Taylor series up to 5 th power is enough to describe state catastrophe of RMS.

$S=a_{0}+a_{1} M+a_{2} M^{2}+a_{3} M^{3}+a_{4} M^{4}+a_{5} M^{5}$

where Srepresents the RMS state; $M$ represents the machine tool state; and $a_{i}(i=0,1, \ldots, 5)$ are a set of data related to RMS state complexity.

Derivation is performed on both sides of formula (15) to obtain the system state's potential function, as shown in formula (16):

$S^{\prime}=a_{1}+2 a_{2} M+3 a_{3} M^{2}+4 a_{4} M^{3}+5 a_{5} M^{4}$

where $S^{\prime}$ represents the potential function of RMS state.

Because the cusp catastrophe model has no cubic coefficient item, formula (16) is transformed. Let $M=n-m$ and $m=a_{4} /\left(5 a_{5}\right)$. The cubic coefficient item in the potential function is eliminated to obtain the standard format of the cusp catastrophe potential function, as shown in formula (17).

$$
\left\{\begin{array}{l}
V=b_{0}+b_{1} n+b_{2} n^{2}+b_{4} n^{4} \\
{\left[\begin{array}{l}
b_{0} \\
b_{1} \\
b_{2} \\
b_{4}
\end{array}\right]=\left[\begin{array}{ccccc}
5 m^{4} & -4 m^{3} & 3 m^{2} & -2 m & 1 \\
-20 m^{3} & 12 m^{2} & -6 m & 2 & 0 \\
30 m^{2} & -12 m & 3 & 0 & 0 \\
5 & 0 & 0 & 0 & 0
\end{array}\right]\left[\begin{array}{l}
a_{5} \\
a_{4} \\
a_{3} \\
a_{2} \\
a_{1}
\end{array}\right]}
\end{array}\right.
$$

where $V$ represents the standard format of cusp catastrophe function; $b_{i}$ are the matrix transformation result of $a_{i}(i=$ $0,1, \ldots, 5)$.

Let $n=\sqrt[4]{\frac{1}{b_{4}}} \theta, b_{4}>0$. This is substituted into formula (17) to obtain the new system state potential function $V$, as shown in formula (18).

$$
\left\{\begin{array}{l}
V=\theta^{4}+u \theta^{2}+v \theta+b_{0} \\
u=\frac{b_{2}}{\sqrt{b_{4}}} \\
v=\frac{b_{1}}{\sqrt[4]{b_{4}}}
\end{array}\right.
$$

where $\theta$ is the RMS state variable and $u$ and $v$ are two control variables in RMS. Based on the previous analysis, the value of the control variable is determined by positive complexity and negative complexity. The value of the control variable is calculated from the ratio of the complexity component with a different effect to overall system complexity. $v$ represents the positive complexity control variable; $u$ represents the negative complexity control variable. Because negative complexity has a negative effect on system stability, the value for the negative complexity control variable is negative, i.e., $u<0 . b_{0}$ is a constant, which does not change the potential function's catastrophe characteristic. Generally, the origin of the potential function is changed to eliminate constant item $b_{0}$. Therefore, the potential function is expressed as formula (19) (Saunders 1980).

$$
\left\{\begin{array}{l}
V=\theta^{4}+u \theta^{2}+v \theta \\
u=-\frac{E_{n}}{E_{X}} \\
v=\frac{E_{p}}{E_{X}}
\end{array}\right.
$$

where $V$ represents RMS's standard format potential function; $\mathrm{u}$ and $\mathrm{v}$ are the control variable of RMS based on information entropy theory.

Derivation is performed on both ends of potential function $V$ in formula (19). The derived function is set to 0 to obtain a balanced surface equation, as shown in formula (20).

$\frac{\partial V}{\partial \theta}=\theta^{3}+u \theta+v=0$

The balanced surface for formula (19) is shown in Fig. 5. This surface comprises 3 lobes in three-dimensional space $(\theta, u, v)$ : top, intermediate and bottom lobes. Among them, the top and bottom lobes are stable, whereas the intermediate lobe is instable. When the phase point $(\theta, u, v)$ reaches the 


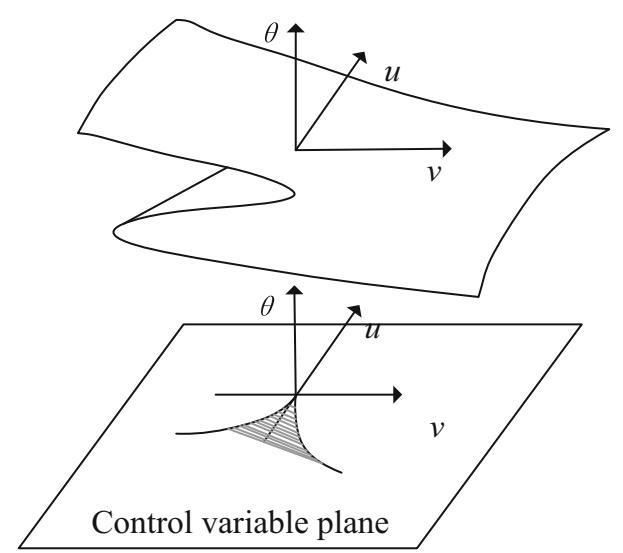

Fig. 5 Balanced surface of cusp catastrophe

edge of the top or bottom lobes, it leaps through the intermediate lobe. Therefore, vertical tangent points on the balanced surface comprise the state catastrophe point set, or singularity set, as shown in formula (21).

$\frac{\partial^{2} V}{\partial \theta^{2}}=3 \theta^{2}+u$

Formulae (20) and (21) are combined to eliminate $\theta$ and obtain the catastrophe characteristic equation, as shown in formula (22).

$\Delta=8 u^{3}+27 v^{2}$

where $\Delta$ represents state catastrophe decision variable of RMS. When $\Delta>0$, there is $27 v^{2}>-8 u^{3}$; i.e., positive complexity is predominant, RMS is in the dynamic stable state and reconfiguration is not needed. When $\Delta=0$, there is $27 v^{2}=-8 u^{3}$. Positive complexity and negative complexity are in a dynamic balanced state; RMS is at a critical point. Slight interference may lead to RMS state catastrophe and the need to reconfigure. When $\Delta<0$, there is $27 v^{2}<-8 u^{3}$. Negative complexity is predominant. RMS stable state catastrophe occurs, and immediate reconfiguration is needed.

\section{Test verification}

An RMS plant in a research institute is used as an example to verify the method proposed in this paper. When there is order change, machine tool failure or processing route change, operations such as adding, rearranging and removing machine tools are required. In this paper, these operations are treated as the processing function and processing capability state change. When the current RMS processing function and processing capability are unable to meet demand, problems such as order delay and system instability arise; RMS reconfiguration is needed to reconfigure the system processing function and processing capability to respond to market fluctuation. The issue of when to implement system reconfiguration has a significant impact on reconfiguration cost, reconfiguration time and production benefit. Therefore, the system state should be analyzed. RMS dynamic complexity variation is analyzed to determine whether system catastrophe occurs and then to decide whether to implement RMS reconfiguration. Therefore, the case study is based on the state data of the machine tool and parts in the RMS plant. Processing function and processing capability variation in an order production process are analyzed. Based on information entropy theory, state probability is converted to system complexity data, which are combined with a cusp catastrophe to analyze the RMS reconfiguration decision. When RMS has a new order or implements a new processing route, this algorithm is reapplied in the system state analysis.

This paper focuses on system-level reconfiguration. In production, different cells are in different states; states of multiple cells comprise the RMS state. Moreover, cross-cell operation in system reconfiguration requires the analysis of multiple cells. Therefore, RMS in this case contains three processing cells. Cell 1 contains four machine tools: $\mathrm{M}_{11}$, $\mathrm{M}_{12}, \mathrm{M}_{3}$ and $\mathrm{M}_{4}$. Cell 2 contains five machine tools: $\mathrm{M}_{3}, \mathrm{M}_{5}$, $\mathrm{M}_{6}, \mathrm{M}_{7}$ and $\mathrm{M}_{8}$. Cell 3 contains three machine tools: $\mathrm{M}_{1 / 2}$, $\mathrm{M}_{5}$ and $\mathrm{M}_{8}$. Among them, $\mathrm{M}_{11}$ and $\mathrm{M}_{12}$ mean that these two machine tools provide processing function 1; machine tool $\mathrm{M}_{1 / 2}$ means that this machine tool provides processing functions 1 and 2 and can switch processing function according to requirements. Other machine tools only provide a single processing function, and each machine tool can only process a single part. The processing rule is first come, first serve. If different processing functions of the same machine tool are required, a part coming late automatically enters the waiting queue. There is one buffer zone before and one after each machine tool; the buffer zone capacity is 5 . The RMS processing function and processing capability are shown in Fig. 6. The order contains six types of parts. The corresponding processing route is planned according to plant processing function and processing capability. The production task is listed in Table 1. In the table, the part number represents the part category; the processing route represents the required processing function codes to complete the processing of the corresponding part; the quantity represents the processing capability required for each part. For instance, part 03 follows processing route $6,7,9$. This means that processing this part requires machine tools $\mathrm{M}_{6}, \mathrm{M}_{7}$ and $\mathrm{M}_{9}$. Based on the processing capability of existing machine tools in the plant, the overall time to process each type of part and the processing time for each step are listed in Table 2. Each line represents the steps and processing time for each part; each column represents the part type processed by each machine tool and the corresponding processing time. Processing task 
Fig. 6 Cell arrangement and machine tool distribution in plant

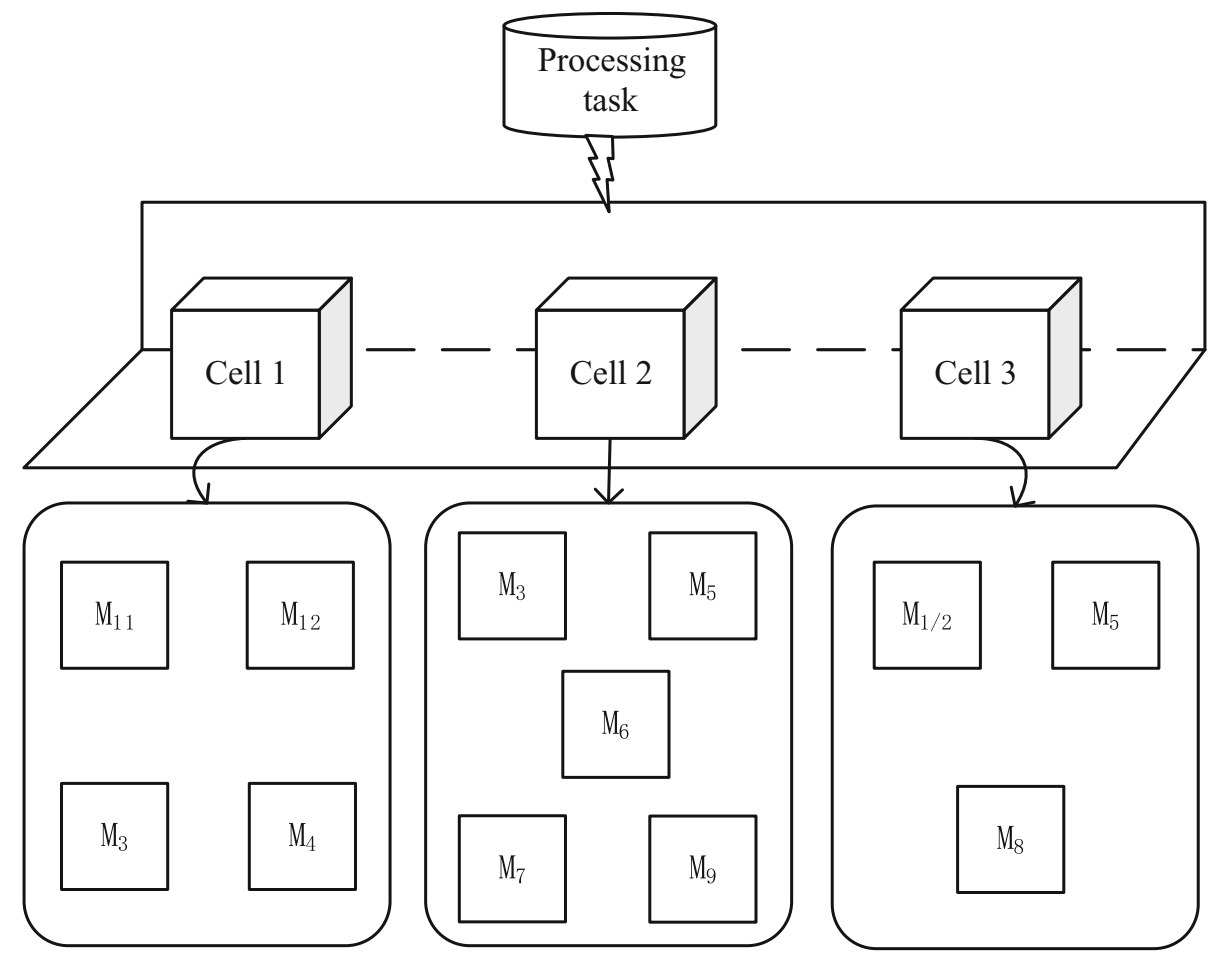

Table 1 Processing task

\begin{tabular}{lll}
\hline Part no. & Processing route & Quantity \\
\hline 01 & 1,3 & 1000 \\
02 & 1,4 & 2000 \\
03 & $6,7,9$ & 2000 \\
04 & $3,5,6,7,9$ & 1000 \\
05 & 1,5 & 1000 \\
06 & 2,8 & 1000 \\
\hline
\end{tabular}

is assigned according to order information. Parts 01 and 02 are processed in cell 1; parts 03 and 04 are processed in cell 2; and parts 05 and 06 are processed in cell 3 . Three processing cells operate simultaneously and the processing in each cell follows the first come, first serve (FCFS) rule.

The data in Table 2 are the fixed procedure time defined in the process design. However, failure in production is random, and the production process in this paper is a stochastic process. Based on the processing time distribution in Table 2, the machine tool and part state in the system are collected every 10 working days and probabilities of the processing function and processing capability are calculated. Because there is a large amount of data, machine tool $\mathrm{M}_{1 / 2}$ is used as an example to demonstrate the probability calculation, which includes the processing function's operative probability $p_{i j k}^{K}$ and inoperative probability $p_{i j k}^{B}$, the failure probability $p_{i j k m}^{G}$, the non-required function processing probability $p_{i j k m}^{\bar{J}}$, the processing capability's non-blocked state probability $p_{i j k}^{C}$ and blocked state probability $p_{i j k}^{D}$, the probability of a previous buffer zone $p_{i j k}^{Q}$, the probability of a subsequent buffer zone $p_{i j k}^{H}$, the machine tool's operative/non-blocked state probability $p_{i j k}^{1}$, the operative/blocked state probability $p_{i j k}^{2}$, and the inoperative probability $p_{i j k}^{3}$, as listed in Table 3 . The machine tool state is monitored and the duration of each state is collected every 10 working days. Machine tool state probabilities $p_{i j k m}^{G}$ and $p_{i j k m}^{\bar{J}}$ are calculated from the ratio of state duration to overall time. Based on formulae (7) and (8), $p_{i j k}^{K}$ and $p_{i j k}^{B}$ are calculated. The throughput of the machine tool in operation is monitored to obtain the average productivity of the corresponding machine tool. Based on formulae (13) and (14), $p_{i j k}^{Q}$ and $p_{i j k}^{H}$, are calculated. Next, based on formulae (9) and (10), $p_{i j k}^{C}$ and $p_{i j k}^{D}$ are calculated.

Based on data statistics and analysis, the operative/nonblocked state probability $p_{i j k}^{1}$, the operative/blocked state probability $p_{i j k}^{2}$, and the inoperative probability $p_{i j k}^{3}$ for each machine tool in each cell are calculated as listed in Tables 4, 5 and 6.

RMS complexity and RMS cusp catastrophe reconfiguration decision data are calculated from the above data via the algorithm proposed in this paper, which includes RMS complexity, positive complexity, negative complexity, decision index and system control variables, as listed in Table 7.

RMS state variation trend diagram in Fig. 7 is based on $\Delta$ data in Table 7. 
Table 2 Single piece processing time for various parts

\begin{tabular}{|c|c|c|c|c|c|c|c|c|c|c|c|c|}
\hline \multirow[t]{3}{*}{ Part no. } & \multicolumn{12}{|c|}{ Processing time $(\mathrm{min})$} \\
\hline & \multicolumn{3}{|l|}{ Cell 1} & \multicolumn{3}{|c|}{ Cell 2} & \multicolumn{6}{|c|}{ Cell 3} \\
\hline & $\mathrm{M}_{11} / \mathrm{M}_{12}$ & $\mathrm{M}_{3}$ & $\mathrm{M}_{4}$ & $\mathrm{M}_{3}$ & $\mathrm{M}_{5}$ & $\mathrm{M}_{6}$ & $\mathrm{M}_{7}$ & $\mathrm{M}_{9}$ & $\mathrm{M}_{1}$ & $\mathrm{M}_{2}$ & $\mathrm{M}_{5}$ & $\mathrm{M}_{8}$ \\
\hline 01 & 2 & 4 & & & & & & & & & & \\
\hline 02 & 3 & & 3 & & & & & & & & & \\
\hline 03 & & & & & & 2 & 2 & 5 & & & & \\
\hline 04 & & & & 3 & 5 & 1 & 2 & 1 & & & & \\
\hline 05 & & & & & & & & & 4 & & 4 & \\
\hline 06 & & & & & & & & & & 3 & & 2 \\
\hline
\end{tabular}

Table 3 Probability analysis for machine tool M1/2

\begin{tabular}{|c|c|c|c|c|c|c|c|c|c|c|c|}
\hline \multirow[t]{3}{*}{ Monitoring time } & \multicolumn{8}{|c|}{ Machine tool state probability } & \multirow[t]{3}{*}{$p_{i j k}^{1}$} & \multirow[t]{3}{*}{$p_{i j k}^{2}$} & \multirow[t]{3}{*}{$p_{i j k}^{3}$} \\
\hline & \multicolumn{2}{|c|}{ Processing function } & \multirow[t]{2}{*}{$p_{i j k}^{K}$} & \multirow[t]{2}{*}{$p_{i j k}^{B}$} & \multicolumn{2}{|c|}{ Processing capability } & \multirow[t]{2}{*}{$p_{i j k}^{C}$} & \multirow[t]{2}{*}{$p_{i j k}^{D}$} & & & \\
\hline & $p_{i j k m}^{G}$ & $p_{i j k m}^{\bar{J}}$ & & & $p_{i j k}^{Q}$ & $p_{i j k}^{H}$ & & & & & \\
\hline 10 & 0.1 & 0.500 & 0.64 & 0.36 & 0.2 & 0.202 & 0.638 & 0.362 & 0.409 & 0.231 & 0.36 \\
\hline 20 & 0.15 & 0.500 & 0.578 & 0.423 & 0.25 & 0.21 & 0.593 & 0.408 & 0.342 & 0.235 & 0.423 \\
\hline 30 & 0.2 & 0.500 & 0.51 & 0.49 & 0.298 & 0.25 & 0.527 & 0.474 & 0.269 & 0.241 & 0.49 \\
\hline 40 & 0.21 & 0.500 & 0.496 & 0.504 & 0.3 & 0.271 & 0.510 & 0.49 & 0.253 & 0.243 & 0.504 \\
\hline 50 & 0.22 & 0.500 & 0.482 & 0.518 & 0.55 & 0.39 & 0.275 & 0.726 & 0.132 & 0.349 & 0.518 \\
\hline 60 & 0.23 & 0.500 & 0.467 & 0.533 & 0.595 & 0.41 & 0.239 & 0.761 & 0.112 & 0.355 & 0.533 \\
\hline
\end{tabular}

Table 4 Probability analysis for machine tool in Cell 1

\begin{tabular}{|c|c|c|c|c|c|c|c|c|c|}
\hline \multirow[t]{2}{*}{ Monitoring time } & \multicolumn{3}{|c|}{$\mathrm{M}_{11} / \mathrm{M}_{12}$} & \multicolumn{3}{|l|}{$\mathrm{M}_{3}$} & \multicolumn{3}{|l|}{$\mathrm{M}_{4}$} \\
\hline & $\overline{p_{i j k}^{1}}$ & $p_{i j k}^{2}$ & $\overline{p_{i j k}^{3}}$ & $\overline{p_{i j k}^{1}}$ & $p_{i j k}^{2}$ & $p_{i j k}^{3}$ & $p_{i j k}^{1}$ & $p_{i j k}^{2}$ & $p_{i j k}^{3}$ \\
\hline 10 & 0.380 & 0.23 & 0.39 & 0.381 & 0.23 & 0.36 & 0.380 & 0.23 & 0.35 \\
\hline 20 & 0.355 & 0.215 & 0.43 & 0.349 & 0.221 & 0.41 & 0.354 & 0.217 & 0.40 \\
\hline 30 & 0.320 & 0.24 & 0.44 & 0.308 & 0.252 & 0.44 & 0.338 & 0.222 & 0.44 \\
\hline 40 & 0.229 & 0.171 & 0.6 & 0.22 & 0.180 & 0.6 & 0.182 & 0.208 & 0.61 \\
\hline 50 & 0.127 & 0.213 & 0.6 & 0.091 & 0.249 & 0.66 & 0.113 & 0.227 & 0.64 \\
\hline 60 & 0.112 & 0.228 & 0.66 & 0.087 & 0.253 & 0.66 & 0.079 & 0.261 & 0.66 \\
\hline
\end{tabular}

Table 5 Probability analysis for machine tool in Cell 2

\begin{tabular}{|c|c|c|c|c|c|c|c|c|c|c|c|c|c|c|c|}
\hline \multirow[t]{2}{*}{ Monitoring time } & \multicolumn{3}{|l|}{$\mathrm{M}_{3}$} & \multicolumn{3}{|l|}{$\mathrm{M}_{5}$} & \multicolumn{3}{|l|}{$\mathrm{M}_{6}$} & \multicolumn{3}{|l|}{$\mathrm{M}_{7}$} & \multicolumn{3}{|l|}{$\mathrm{M}_{9}$} \\
\hline & $\overline{p_{i j k}^{1}}$ & $p_{i j k}^{2}$ & $p_{i j k}^{3}$ & $\overline{p_{i j k}^{1}}$ & $p_{i j k}^{2}$ & $\overline{p_{i j k}^{3}}$ & $\overline{p_{i j k}^{1}}$ & $p_{i j k}^{2}$ & $p_{i j k}^{3}$ & $\overline{p_{i j k}^{1}}$ & $p_{i j k}^{2}$ & $p_{i j k}^{3}$ & $p_{i j k}^{1}$ & $p_{i j k}^{2}$ & $p_{i j k}^{3}$ \\
\hline 10 & 0.403 & 0.297 & 0.31 & 0.429 & 0.27 & 0.3 & 0.379 & 0.23 & 0.39 & 0.504 & 0.196 & 0.33 & 0.384 & 0.225 & 0.38 \\
\hline 20 & 0.331 & 0.269 & 0.4 & 0.328 & 0.271 & 0.41 & 0.347 & 0.223 & 0.43 & 0.422 & 0.177 & 0.4 & 0.41 & 0.159 & 0.43 \\
\hline 30 & 0.316 & 0.274 & 0.41 & 0.296 & 0.293 & 0.41 & 0.288 & 0.271 & 0.44 & 0.351 & 0.238 & 0.41 & 0.323 & 0.236 & 0.44 \\
\hline 40 & 0.185 & 0.215 & 0.6 & 0.174 & 0.225 & 0.6 & 0.232 & 0.167 & 0.6 & 0.235 & 0.164 & 0.6 & 0.238 & 0.162 & 0.6 \\
\hline 50 & 0.119 & 0.221 & 0.66 & 0.114 & 0.225 & 0.66 & 0.108 & 0.231 & 0.66 & 0.115 & 0.244 & 0.64 & 0.19 & 0.149 & 0.65 \\
\hline 60 & 0.088 & 0.252 & 0.66 & 0.09 & 0.249 & 0.66 & 0.092 & 0.247 & 0.66 & 0.081 & 0.258 & 0.66 & 0.158 & 0.171 & 0.67 \\
\hline
\end{tabular}

The system state trend diagram in Fig. 7 shows the trend of system state detection index $\Delta$ : after a relatively stable production period, the system experiences a state catastrophe, which triggers system reconfiguration. In the first 40 working days, when $\Delta$ is above 0 and stays around 0.8 , fluctuation is relatively small. This shows that the system is in a stable operation period and reconfiguration is not needed. During the 40 th $\sim 50$ th working days, $\Delta$ decreases dramat- 
Table 6 Probability analysis for machine tool in Cell 3

\begin{tabular}{|c|c|c|c|c|c|c|c|c|c|c|c|c|}
\hline \multirow[t]{2}{*}{ Monitoring time } & \multicolumn{3}{|l|}{$\underline{\mathrm{M}_{1}}$} & \multicolumn{3}{|l|}{$\underline{\mathrm{M}_{2}}$} & \multicolumn{3}{|l|}{$\mathrm{M}_{5}$} & \multicolumn{3}{|l|}{$\mathrm{M}_{8}$} \\
\hline & $\overline{p_{i j k}^{1}}$ & $p_{i j k}^{2}$ & $p_{i j k}^{3}$ & $\overline{p_{i j k}^{1}}$ & $p_{i j k}^{2}$ & $p_{i j k}^{3}$ & $\overline{p_{i j k}^{1}}$ & $p_{i j k}^{2}$ & $p_{i j k}^{3}$ & $\overline{p_{i j k}^{1}}$ & $p_{i j k}^{2}$ & $\overline{p_{i j k}^{3}}$ \\
\hline 10 & 0.409 & 0.231 & 0.36 & 0.409 & 0.231 & 0.36 & 0.393 & 0.346 & 0.261 & 0.204 & 0.2 & 0.36 \\
\hline 20 & 0.342 & 0.235 & 0.423 & 0.342 & 0.235 & 0.423 & 0.48 & 0.290 & 0.23 & 0.336 & 0.264 & 0.4 \\
\hline 30 & 0.269 & 0.241 & 0.49 & 0.269 & 0.241 & 0.49 & 0.430 & 0.33 & 0.24 & 0.330 & 0.26 & 0.41 \\
\hline 40 & 0.253 & 0.243 & 0.504 & 0.253 & 0.243 & 0.504 & 0.448 & 0.352 & 0.2 & 0.392 & 0.308 & 0.5 \\
\hline 50 & 0.132 & 0.349 & 0.518 & 0.132 & 0.349 & 0.518 & 0.215 & 0.495 & 0.29 & 0.12 & 0.22 & 0.6 \\
\hline 60 & 0.112 & 0.355 & 0.533 & 0.112 & 0.355 & 0.533 & 0.175 & 0.525 & 0.3 & 0.081 & 0.259 & 0.66 \\
\hline
\end{tabular}

Table 7 Complexity and reconfiguration decision data

\begin{tabular}{|c|c|c|c|c|c|c|}
\hline \multirow[t]{2}{*}{ Monitoring time } & \multicolumn{3}{|c|}{ Complexity } & \multicolumn{3}{|c|}{ Reconfiguration decision } \\
\hline & $E_{X}$ & $E_{p}$ & $E_{n}$ & $\Delta$ & $v$ & $u$ \\
\hline 10 & 15.34 & 5.28 & 10.06 & 0.942 & 0.344 & -0.656 \\
\hline 20 & 16.86 & 5.79 & 11.07 & 0.920 & 0.343 & -0.657 \\
\hline 30 & 16.98 & 5.78 & 11.20 & 0.833 & 0.340 & -0.660 \\
\hline 40 & 17.00 & 5.75 & 11.25 & 0.770 & 0.338 & -0.662 \\
\hline 50 & 14.09 & 4.17 & 9.92 & -0.427 & 0.296 & -0.704 \\
\hline 60 & 13.71 & 3.70 & 10.01 & -1.147 & 0.270 & -0.730 \\
\hline
\end{tabular}

RMS state catastrophe is the best time to implement RMS reconfiguration. Next, based on information entropy theory, a system complexity quantitative model is created to analyze the effect of the system complexity component on the system state. That is then combined with the cusp catastrophe theory to analyze system state variation under the effect of various complexity components. The cusp catastrophe determinant condition helps identify the state catastrophe point and decide the RMS reconfiguration point. The test shows that information entropy-based system complexity analysis provides a quantitative description of system complexity. System complexity variation is analyzed in terms of processing function and processing capability to reveal the nature of the system state. Application of the cusp catastrophe helps identify the system state catastrophe moment. This provides evidence for the decision maker to decide the reconfiguration point, promptly implement system reconfiguration and maintain RMS vitality. Subsequent work focuses on an indepth investigation of the RMS state catastrophe mechanism, which includes an analysis of the production factors that lead to system state catastrophe.

Acknowledgements The authors are grateful to the anonymous reviewers for their comments, which have helped to improve this paper.

Funding The National Natural Science Foundation, China (Project No. 51105039).

\section{Compliance with ethical standards}

Conflict of interest The authors declare that they have no conflict of interest. 
Open Access This article is distributed under the terms of the Creative Commons Attribution 4.0 International License (http://creativecomm ons.org/licenses/by/4.0/), which permits unrestricted use, distribution, and reproduction in any medium, provided you give appropriate credit to the original author(s) and the source, provide a link to the Creative Commons license, and indicate if changes were made.

\section{References}

Barunik, J., \& Vosvrda, M. (2009). Can a stochastic cusp catastrophe model explain stock market crashes? Journal of Economic Dynamics and Control, 33(10), 1824-1836.

Bi, Z. M., Lang, S. Y. T., Shen, W., \& Wang, L. (2008). Reconfigurable manufacturing systems: The state of the art. International Journal of Production Research, 46(4), 967-992.

Cai, Z.-Y. (2004). Performance measurement of reconfigurable manufacturing system. Acta Aeronautica et Astronautica Sinica, 25(5), 516-519.

Chow, P. T., Cheung, S. O., \& Yiu, T. W. (2012). A cusp catastrophe model of withdrawal in construction project dispute negotiation. Automation in Construction, 22, 597-604.

Czinner, V. G., \& Mena, F. C. (2016). Relative information entropy in cosmology: The problem of information entanglement. Physics Letters B, 758, 9-13.

Dou, W., \& Ghose, S. (2006). A dynamic nonlinear model of online retail competition using cusp catastrophe theory. Journal of Business Research, 59(7), 838-848.

Duan, J. (2012). Structural complexity modeling and evaluation of manufacturing systems based on state entropy. Journal of Mechanical Engineering, 48(05), 92-100.

Goyal, K. K., Jain, P. K., \& Jain, M. (2012). Optimal configuration selection for reconfigurable manufacturing system using NSGA II and TOPSIS. International Journal of Production Research, 50(15), 4175-4191.

Goyal, K. K., Jain, P. K., \& Jain, M. (2013). A novel methodology to measure the responsiveness of RMTs in reconfigurable manufacturing system. Journal of Manufacturing Systems, 32(4), 724-730.

Gupta, A., Jain, P. K., \& Kumar, D. (2013). A novel approach for part family formation for reconfiguration manufacturing system. Opsearch, 51(1), 76-97.

Hasan, F., Jain, P. K., \& Kumar, D. (2014). Service level as performance index for reconfigurable manufacturing system involving multiple part families. Procedia Engineering, 69, 814-821.

High-Tech Strategy 2020. (2010). Power games in space: The German high-tech strategy and European space policy. Berlin: The German Federal Government.

Hu, B., \& Xia, N. (2015). Cusp catastrophe model for sudden changes in a person's behavior. Information Sciences, 294, 489-512.

Koren, Y. (2013). The rapid responsiveness of RMS. International Journal of Production Research, 51(23-24), 6817-6827.

Koren, Y., Heisel, U., Jovane, F., Moriwaki, T., Pritschow, G., Ulsoy, G., et al. (1999). Reconfigurable Manufacturing Systems. CIRP Annals: Manufacturing Technology, 48(2), 527-540.

Lee, J., Bagheri, B., Kao, H. A., \& Lapira, E. (2015). Industry 4.0 and manufacturing transformation. Manufacturing Leadership Journal, 2, 2-9.

Mehrabi, M. G., Ulsoy, A. G., \& Koren, Y. (2000a). Reconfigurable manufacturing systems: Key to future manufacturing. Journal of Intelligent Manufacturing, 11(4), 403-419.

Mehrabi, M. G., Ulsoy, A. G., \& Koren, Y. (2000b). Reconfigurable manufacturing systems and their enabling technologies. International Journal of Manufacturing Technology and Management, 1(1), 113-130.
Mehrabi, M. G., Ulsoy, A. G., Koren, Y., \& Heytler, P. (2002). Trends and perspectives in flexible and reconfigurable manufacturing systems. Journal of Intelligent Manufacturing, 13(2), 135-146.

Qin, L. (2004). Complexity and stability in the forest communities. Guangdong: Sun Yat-sen University.

Rao, Y. (2006). Entropy-based measurement of manufacturing system complexity and its application in scheduling. Chinese Journal of Mechanical Engineering, 42(07), 8-13.

Renzi, C., Leali, F., Cavazzuti, M., \& Andrisano, A. O. (2014). A review on artificial intelligence applications to the optimal design of dedicated and reconfigurable manufacturing systems. The International Journal of Advanced Manufacturing Technology, 72(1-4), 403-418.

Rösiö, C., \& Säfsten, K. (2013). Reconfigurable production system design-Theoretical and practical challenges. Journal of Manufacturing Technology Management, 24(7), 998-1018.

Saunders, P. T. (1980). An introduction to catastrophe theory. Cambridge: Cambridge University Press.

Shannon, C. E. (1948). IEEE Xplore abstract-A mathematical theory of communication. Bell System Technical Journal, 27(3), 379-423.

Sethi, V., \& King, R. C. (1998). An application of the cusp catastrophe model to user information satisfaction. Information \& Management, 34(1), 41-53.

Smart, J., Calinescu, A., \& Huatuco, L. H. (2013). Extending the information-theoretic measures of the dynamic complexity of manufacturing systems. International Journal of Production Research, 51(2), 362-379.

Thom, R., Benjamin, W. A., \& Reading, M. A. (1975). Structural stability and morphogenesis. Reading, MA: W.A. Benjamin.

Tsujimura, Y., \& Gen, M. (1999). Parts loading scheduling in a flexible forging machine using an advanced genetic algorithm. Journal of Intelligent Manufacturing, 10(2), 149-159.

Wang, C. (2000). On the possibility of manufacturing system reconfiguration. Computer Integrated Manufacturing Systems, 6(4), 1-5.

Wang, G. X., Huang, S. H., Shang, X. W., Yan, Y., \& Du, J. J. (2016a). Formation of part family for reconfigurable manufacturing systems considering bypassing moves and idle machines. Journal of Manufacturing Systems, 41, 120-129.

Wang, G. X., Huang, S. H., Yan, Y., \& Du, J. J. (2016b). Reconfiguration schemes evaluation based on preference ranking of key characteristics of reconfigurable manufacturing systems. International Journal of Advanced Manufacturing Technology, 89(5), 22312249.

Wu, Z. J., Ning, R. X., \& Wang, A. M. (2007). Grey fuzzy synthetically evaluation method for RMS layout planning. China Mechanical Engineering, 18(19), 2313-2318.

Zhang, Z. (2011). Dissipative structure models of manufacturing systems and its measurement based on cusp catastrophe. Journal of Mechanical Engineering, 47(14), 192-198.

Zhao, X., Wang, J., \& Luo, Z. (2000a). A stochastic model of a reconfigurable manufacturing system part 1: A framework. International Journal of Production Research, 38(10), 2273-2285.

Zhao, X., Wang, J., \& Luo, Z. (2000b). A stochastic model of a reconfigurable manufacturing system part 2: Optimal configurations. International Journal of Production Research, 38(12), 2829-2842.

Zhao, X., Wang, J., \& Luo, Z. (2001a). A stochastic model of a reconfigurable manufacturing system part 3: Optimal selection policy. International Journal of Production Research, 39(4), 747-758.

Zhao, X., Wang, J., \& Luo, Z. (2001b). A stochastic model of a reconfigurable manufacturing system-Part 4: Performance measure. International Journal of Production Research, 39(6), 1113-1126. 